\title{
Discrimination of temperature and strain by combined refractive index and birefringence measurements using coherent Rayleigh sensing
}

\author{
Xin Lu*, Marcelo A. Soto, Luc Thévenaz \\ EPFL Swiss Federal Institute of Technology, Institute of Electrical Engineering, \\ SCI STI LT, Station 11, CH-1015 Lausanne, Switzerland \\ *E-mail: xin.lu@epfl.ch
}

\begin{abstract}
Temperature and strain discrimination is experimentally demonstrated in an elliptical-core polarization-maintaining fiber by making use of Rayleigh-based distributed birefringence measurements and the frequency shift of the correlation peak obtained by standard coherent optical time-domain reflectometry. The high sensitivity of coherent Rayleigh sensing and the very distinct behavior of birefringence makes the two quantities clearly discriminated, resulting in temperature and strain accuracies of $\sim 40 \mathrm{mK}$ and $\sim 0.5 \mu \varepsilon$, respectively, for distributed measurements with a $2 \mathrm{~m}$ spatial resolution.
\end{abstract}

Keywords: Fiber optics sensors, distributed fiber sensing, birefringence, coherent Rayleigh scattering.

\section{INTRODUCTION}

Distributed fiber sensors (DFS) based on different scattering mechanisms have been proposed over three decades and successfully commercialized to continuously measure environmental variables, such as temperature and $\operatorname{strain}^{1}$. The Raman sensor is sensitive only to temperature, which is retrieved from the ratio between optical power of anti-Stokes and Stokes/Rayleigh waves. On the other hand, strain and/or temperature variations can be obtained from the Brillouin frequency shift in a Brillouin sensor or the cross-correlation spectral shift in a DFS based on coherent Rayleigh scattering. The optical fiber used in Rayleigh sensing can be seen as a weak fiber Bragg grating (FBG) with random pitch, making the coherent Rayleigh sensor potentially as sensitive as a FBG sensor. This means that Rayleigh-based sensors are threeorder of magnitude more sensitive than Brillouin sensors ${ }^{2}$, and can provide better measurand resolution compared to Raman sensors; however, they suffer from the temperature-strain cross-sensitivity issues.

As the distributed sensing technique keeps progressing, its application area has been broadened and many other physical quantities can be measured by DFS. Thus, for instance, the phase birefringence profile along polarization-maintaining (PM) fibers can be retrieved by dynamic Brillouin gratings (DBG), optical frequency-domain reflectometry (OFDR) and coherent optical time-domain reflectometry $(\mathrm{COTDR})^{3-5}$. Since the birefringence is also dependent on environmental variations, all methods for birefringence measurements can be used for distributed temperature and strain sensing, leading to sensitivities one order of magnitude higher than in a Brillouin sensor ${ }^{3}$. In addition, cross-sensitivity issues between temperature and strain have been mitigated by combining a birefringence measurement with a standard Brillouin or OFDR sensing measurement ${ }^{3-4}$. However, the generation of DBG requires three high power lightwaves with a precise optical frequency control, whilst OFDR provides a larger measurement error compared to a COTDR sensor due to its broad correlation peak ${ }^{6}$, and the sensing distance is usually limited by the coherent length of the light source.

In this paper, the discrimination of temperature and strain is experimentally realized by simultaneously measuring the birefringence and the refractive index changes using a COTDR technique along an elliptical-core PM fiber. This method requires moderate optical power and access to only one fiber end, and can achieve a much longer sensing distance. Moreover, the proposed technique has the potential to better discriminate the quantities and provide higher resolution than the reported results due to the high sensitivity of COTDR sensors. The limit of the discrimination method based on birefringence measurement is experimentally investigated by applying small temperature and strain changes.

\section{EXPERIMENT}

According to the first proposed birefringence measurement using COTDR technique, coherent Rayleigh backscattered traces are acquired successively from the two orthogonal axes of the PM fiber, and then information on birefringence is retrieved by cross-correlating the traces from the $2 \mathrm{axes}^{5}$. Hence, this technique works essentially like a conventional 
COTDR sensor. The high sensitivity of the coherent Rayleigh sensor may potentially introduce large measurement errors as a result of small environmental perturbations between the acquisitions of the two sets of Rayleigh traces. Although this error is fairly small compared to the absolute value of birefringence, it can no longer be neglected in sensing measurements. It would be therefore much beneficial if the traces from both axes can be acquired simultaneously to mitigate this

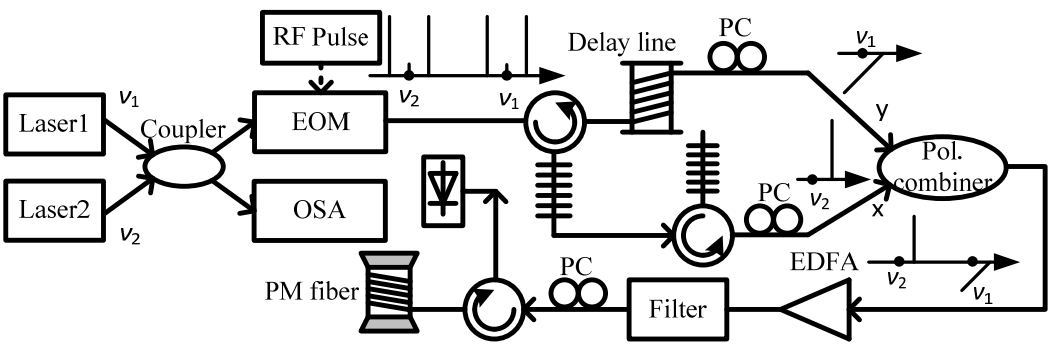

Figure 1. Implemented experimental setup for single shot acquisition of the Rayleigh traces from the two polarization axes. environmental perturbation. The experimental setup shown in Fig. 1 can meet this requirement, making it fully appropriate to discriminate temperature and $\operatorname{strain}^{7}$.

Two lasers operating at $\sim 1534 \mathrm{~nm}$ are employed for the 2 orthogonal axes of a PM fiber with a frequency difference that roughly matches the birefringence of the fiber. The continuous waves from the lasers are first coupled together, then one part of the combined light enters a high-resolution $(5 \mathrm{MHz})$ optical spectrum analyzer (OSA) to monitor the frequency difference and the other part passes through an electro-optic modulator (EOM) driven by RF pulses. In this way, the frequency scanning and pulse shaping are realized simultaneously. The pulse width is $20 \mathrm{~ns}$, corresponding to a $2 \mathrm{~m}$ spatial resolution, limited by the modulation bandwidth of the microwave generator. The generated pulses at preset frequencies are selected by two narrow FBGs, and a time offset between the selected pulses is realized by inserting a delay line in one of the branches. In this experiment, a $640-\mathrm{m}$ long fiber is used, corresponding to a $\sim 3.4 \mu$ s time delay which must fulfil the condition to be longer than the return trip propagation time in the sensing fiber. The impact of the environmental perturbations can be fully neglected within such a short time. The polarizations of the optical pulses at different frequencies are adjusted by polarization controllers (PC), so that the pulses are re-combined orthogonally. Then, an erbium-doped fiber amplifier (EDFA) is used to boost the pulse power, whilst the amplified spontaneous emission (ASE) is suppressed by a broadband bandpass filter. A third PC is used to make sure that the amplified pulses are launched into the correct axes of the sensing fiber. A $97 \mathrm{~m}$-long elliptical-core PM fiber is employed in this experiment with an average birefringence of $\sim 3.26 \times 10^{-4}$. Finally, the Rayleigh traces from each axis are acquired by a $125 \mathrm{MHz}$ detector and are observed temporally separated by the fixed optical time delay through a single shot acquisition.

The standard COTDR sensing can be realized by cross-correlating the Rayleigh traces from the same axis obtained in successive measurements, so that the calculated frequency shift $\Delta v_{\text {COTDR }}$ can represent the temperature and strain changes. On the other hand, the birefringence along the fiber is retrieved by the cross-correlation of the Rayleigh traces from the orthogonal axes in the same measurement. The environmental variations between the birefringence measurements introduce a difference $\Delta v_{\text {bire }}$ of the corresponding frequency shift. Since both temperature and strain contributes to the frequency shift, the combined effect obtained by the two sensing methods is expressed as:

$$
\left[\begin{array}{c}
\Delta v_{\text {COTDR }} \\
\Delta v_{\text {bire }}
\end{array}\right]=\left[\begin{array}{cc}
S_{T, \text { COTDR }} & S_{\varepsilon, \text { COTDR }} \\
S_{T, \text { bire }} & S_{\varepsilon, \text { bire }}
\end{array}\right]\left[\begin{array}{c}
\Delta T \\
\Delta \varepsilon
\end{array}\right],
$$

where $\Delta T$ and $\Delta \varepsilon$ are the temperature and strain changes, $S_{T, C O T D R}$ and $S_{\varepsilon, \text { COTDR }}$ are the temperature and strain sensitivities of the COTDR sensor, $S_{T, b i r e}$ and $S_{\varepsilon, \text { bire }}$ represent the temperature and strain sensitivities of the fiber birefringence, respectively. As a result, the temperature and strain can be discriminated by the measured $\Delta v_{\text {COTDR }}$ and $\Delta v_{\text {bire }}{ }^{3}$ :

$$
\left[\begin{array}{c}
\Delta T \\
\Delta \varepsilon
\end{array}\right]=\frac{1}{S_{T, \text { COTDR }} S_{\varepsilon, \text { bire }}-S_{\varepsilon, \text { COTDR }} S_{T, \text { bire }}}\left[\begin{array}{cc}
S_{\varepsilon, \text { bire }} & -S_{\varepsilon, \text { COTDR }} \\
-S_{T, \text { bire }} & S_{T, \text { COTDR }}
\end{array}\right]\left[\begin{array}{c}
\Delta v_{\text {COTDR }} \\
\Delta v_{\text {bire }}
\end{array}\right] .
$$

Hence, the resolution of the discriminated temperature $\delta_{\Delta \mathrm{T}}$ and strain $\delta_{\Delta \varepsilon}$ can be obtained from Eq. (2)

$$
\begin{aligned}
& \sigma_{\Delta T}=\sqrt{\left(S_{\varepsilon, \text { bire }} \cdot \sigma_{\Delta v, \text { COTDR }}\right)^{2}+\left(S_{\varepsilon, \text { COTDR }} \cdot \sigma_{\Delta v, \text { bire }}\right)^{2}} /\left|S_{T, \text { COTDR }} \cdot S_{\varepsilon, \text { bire }}-S_{\varepsilon, \text { COTDR }} \cdot S_{T, \text { bire }}\right|, \\
& \sigma_{\Delta \varepsilon}=\sqrt{\left(S_{T, \text { bire }} \cdot \sigma_{\Delta v, \text { COTDR }}\right)^{2}+\left(S_{T, \text { COTDR }} \cdot \sigma_{\Delta v, \text { bire }}\right)^{2}} /\left|S_{T, \text { COTDR }} \cdot S_{\varepsilon, \text { bire }}-S_{\varepsilon, \text { COTDR }} \cdot S_{T, \text { bire }}\right|,
\end{aligned}
$$


where $\sigma_{\Delta v, \text { COTDR }}$ and $\sigma_{\Delta v \text {,bire }}$ represent the standard deviation of the frequency shift obtained by COTDR sensor and by the birefringence measurement, respectively.

\section{EXPERIMENTAL RESULTS AND DISCUSSION}

To demonstrate the discrimination of temperature and strain, the birefringence profile of the PM fiber is measured twice successively by the experimental setup shown in Fig. 1, while a $1.9 \mathrm{~K}$ temperature change is applied to a fiber segment located between $84 \mathrm{~m}$ and $88 \mathrm{~m}$ and the last $2.1 \mathrm{~m}$ of the fiber is attached to a moving stage to distinctively apply a strain change of $25.5 \mu \varepsilon$. The high sensitivity of COTDR sensing requires a $9 \mathrm{GHz}$ frequency scanning range by $10 \mathrm{MHz}$ steps.
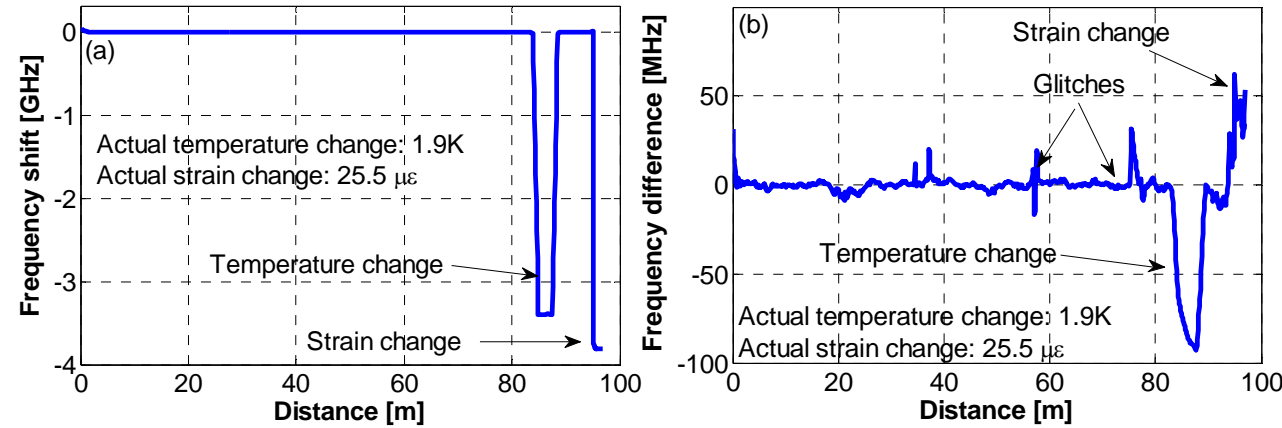

Figure 2. (a) Frequency shift obtained from a standard COTDR sensor and (b) frequency difference of birefringence measurements for an elliptical-core PM fiber.

The frequency shift of the correlation peak measured in the standard COTDR along the slow axis of the PM fiber is shown in Fig. 2(a). Due to the high sensitivity of the Rayleigh measurements and the large number of scanned frequencies, a high-amplitude correlation peak is obtained along the fiber, thus leading to a very clean trace in Fig. 2(a), which shows two sharp frequency shifts at the heated and strained sections. On the other hand, the birefringence profile is retrieved by the cross-correlation of the traces from the orthogonal axes, which leads to a correlation peak of lower amplitude and broadened by random birefringence fluctuations. Thus, measurements with lower contrast are obtained in this case, making birefringence measurements more vulnerable to noise. As a result, a noisier trace of the frequency difference between two birefringence measurements is shown in Fig. 2(b). Note that some random peaks are observed in the figure outside the strained and heated fiber sections, which can be explained by local fast longitudinal birefringence changes within the spatial resolution. In addition, it should be also noted that the obtained curve shows a broad peak at the hot-spot position; this is because the frequency shift due to the thermally-induced birefringence change is very small as a result of the small local temperature increase of $1.9 \mathrm{~K}$.
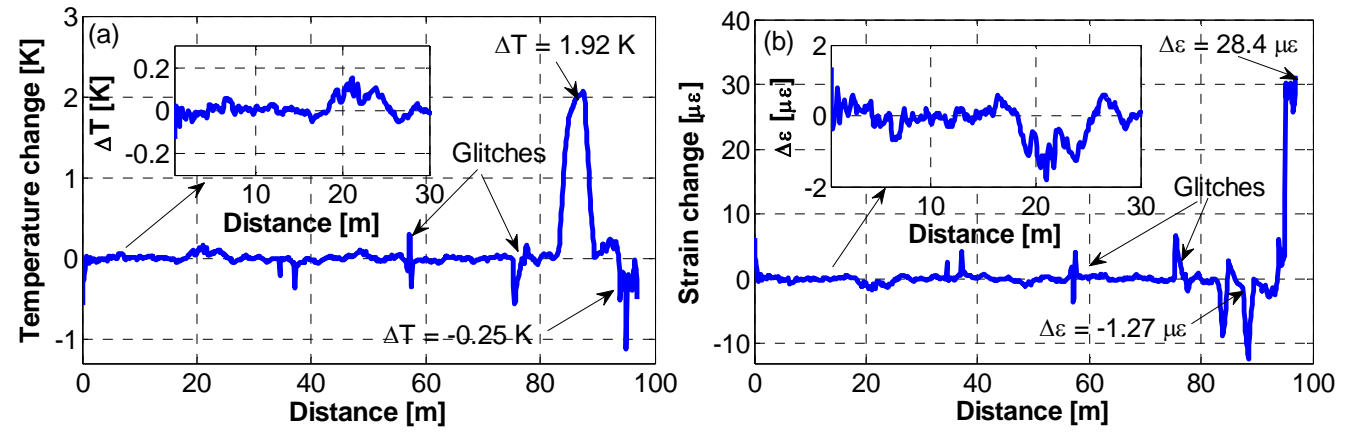

Figure 3. Retrieved (a) temperature and (b) strain profiles along the PM fiber, and the insets are the enlarged view of the first $30 \mathrm{~m}$.

The temperature and strain sensitivities for both COTDR and birefringence are experimentally determined for the used elliptical-core PM fiber, resulting in $S_{T, \text { COTDR }}=-1.739 \mathrm{GHz} / \mathrm{K}, S_{\varepsilon, \text { COTDR }}=-150 \mathrm{MHz} / \mu \varepsilon, S_{T, \text { bire }}=-43.8 \mathrm{MHz} / \mathrm{K}$ and $S_{\varepsilon, b i r e}=1 \mathrm{MHz} / \mu \varepsilon$, respectively. Inserting those parameters into Eq. (2), the temperature and strain values can be calculated from the measurements of $\Delta v_{\text {COTDR }}$ and $\Delta v_{\text {bire }}$ shown in Fig. 2. The retrieved temperature and strain profiles along the fiber are presented in Fig. 3. The measured average temperature $(1.92 \mathrm{~K})$ and strain $(28.4 \mu \varepsilon)$ changes at corresponding heated and strained sections are very close to the actual applied values, validating the proposed technique 
for temperature-strain discrimination. Only a minor temperature change of $-0.25 \mathrm{~K}$ is obtained in the temperature profile at the strained section, and a strain variation of $-1.3 \mu \varepsilon$ leaks into the strain profile at the transitions of the heated section.

As shown in Fig. 3, the obtained temperature and strain profiles exhibit variations similar to the frequency fluctuations obtained from the birefringence measurement, demonstrating the high impact of the birefringence measurement on the final accuracy of the proposed method, which may be explained by the corresponding lower sensitivity compared to the COTDR sensing technique. Some spurious and sharp peaks (of up to $\sim 1 \mathrm{~K}$ and $\sim 10 \mu \varepsilon$ in the worst case) are also observed in Fig. 3 due to their presence in the birefringence measurement, demonstrating the limit of the discrimination method, It should be noted that previous measurements based on OFDR and BDG technique report no apparent random peaks because of the larger temperature and strain changes applied in the experiments ${ }^{3-4}$ compared to our case.

Despite the mentioned spurious peaks, the proposed method provides more reliable temperature-strain discrimination and better measurements compared to other techniques due to the higher sensitivity of the COTDR interrogation. For example, the insets of Fig. 3 show measurand resolutions of $\sim 40 \mathrm{mK}$ and $\sim 0.5 \mu \varepsilon$ for the first $30 \mathrm{~m}$ of the fiber, which are more accurate than the reported measurements by OFDR and DBG techniques. Furthermore it should be mentioned that experimental results agree well with the theoretical results calculated from Eqs. (3) and (4), with $\sigma_{\Delta v, \mathrm{COTDR}}=2.95 \mathrm{MHz}$ and $\sigma_{\Delta v \text {,bire }}=2.19 \mathrm{MHz}$ obtained from Fig. 2. To reduce the error in the discrimination, a small conditional number is desired ${ }^{9}$. The number associated with Eq. (2) is calculated to be $\sim 367.9$ based on the temperature and strain sensitivities of the COTDR technique and birefringence measurement, which is $\sim 3$ times smaller than the conditional number ( 989.7) using a DBG scheme ${ }^{3}$. Thus the matrix in Eq. (2) is better conditioned for the Rayleigh method here proposed.

\section{CONCLUSION}

In this paper a method for temperature-strain discrimination in distributed fiber sensing has been proposed and experimentally demonstrated in an elliptic-core PM fiber using standard COTDR and Rayleigh-based birefringence measurements. The proposed method provides a more reliable and accurate technique to discriminate temperature and strain when compared to DBG, though based on a similar principle. This is basically due to the higher sensitivity of the COTDR interrogation technique (when compared to a conventional Brillouin and DBG sensing), which leads to a better conditioned matrix representing the measurement technique. It has been however found out that the limitation of the method is basically given by to the lower sensitivity of the birefringence, which has led to traces containing unwanted glitches. Further research is still required to eliminate those glitches and achieve a more accurate birefringence profile.

The authors thank the support from the European Space Agency (ESA) in the framework of the Call of Order 09 - EPFLESA / Contract 4000108465 and the Swiss Commission for Technology and Innovation (Project 18337.2 PFNM-NM).

\section{REFERENCES}

[1] Bao, X. and Chen, L., "Recent progress in distributed fiber optic sensors," Sensors 12(7), 8601-8639 (2012).

[2] Lu, X., Soto, M. A. and Thévenaz, L., "MilliKelvin resolution in cryogenic temperature distributed fibre sensing based on coherent Rayleigh scattering," Proc. SPIE 9157, 91573R (2014).

[3] Zou, W., He, Z. and Hotate, K., "Demonstration of Brillouin distributed discrimination of strain and temperature using a polarization-maintaining optical fiber," IEEE Photon. Technol. Lett., 22(8), 526-528 (2010).

[4] Li, W., Chen, L. and Bao, X., "Compensation of temperature and strain coefficients due to local birefringence using optical frequency domain reflectometry," Opt. Commun., 311, 26-32 (2013).

[5] Soto, M. A., Lu, X., Martins, H. F., et al., "Distributed phase birefringence measurements based on polarization correlation in phase-sensitive optical time-domain reflectometers," Opt. Express 23(19), 24923-24936 (2015).

[6] Du, Y. Liu, T., Ding, Z., Han, Q., Liu, K., Jiang, J., et al., "Cryogenic Temperature Measurement Using Rayleigh Backscattering Spectra Shift by OFDR," IEEE Photon. Technol. Lett., 26(11), 1150-1153 (2014).

[7] Lu, X., Soto, M. A. and Thévenaz, L., "Novel technique for distributed fibre sensing based on coherent Rayleigh scattering measurements of birefringence," Proc. SPIE 9916, 991633 (2016).

[8] Taylor, J. R., [An Introduction to Error Analysis], University Science Books, Sausalito, 79 (1997).

[9] Yoon, H.-J., Costantini, D. M., Limberger, et al., "In situ strain and temperature monitoring of adaptive composite materials," J. Intell. Mater. Syst. Struct. 17(12), 1059-1067 (2006). 